\title{
A Novel Otsu Method Based on Prior Area Information for Concealed Target Detection in PMMW Images
}

\author{
Jintao Xiong, Simeng Li, Jianyu Yang, Xiaopeng Xue, Jingqi Mao and Zenan Mao \\ University of Electronic Science and Technology of China, Chengdu 611731, Sichuan, P. R. China
}

\begin{abstract}
A novel double-threshold Otsu algorithm based on prior area is proposed to improve the detection accuracy of small target in this paper. The proposed algorithm makes good use of the area information of each region in images to improve the accuracy of target detection, and can handle scenes containing small target which has low computational complexity, and achieves robust detection for concealed target in Passive Millimeter Wave (PMMW) images. Experiments are conducted on PMMW images collected by PMMW imaging system. The experimental results show that the proposed algorithm can effectively detect the concealed targets, and has better performance in target detection than other traditional threshold segmentation algorithm based on Otsu.
\end{abstract}

\section{Introduction}

PMMW imaging system obtain image through detecting the difference of millimeter energy that objects radiate, which has great performance in penetrating [1]. It not only can detect metal objects hidden in the fabric, but also can detect plastic pistols, explosives and other dangerous objects. These features make it ideal for battlefield reconnaissance and security of important places. Target detection is an important component of its application.

Detection has been studied for more than a decade, there are many detection methods such as target detection algorithm based on k-means[2], target detection algorithm based on mixed gauss model[3]-[5] and target detection algorithm based on active contour model[2], [6] and so on. These algorithms can meet the testing requirements of the PMMW images, but are computationally costly, cannot achieve the requirements of real-time detection. The target detection algorithm based on Otsu [7]-[8] method are widely applied in human image processing systems. It has simple principle, a small amount of calculation and stable performance in the detection process. But it will lose the small target. In order to solve this problem, we propose a novel Otsu algorithm based on prior area for target detection in PMMW images. The algorithm modifies the weighting coefficients in the objective function according to the prior area information of different regions of the PMMW images, obtain more accurate threshold and improve the accuracy in target detection. The experimental results show that the algorithm proposed in this paper has good effect, and meet the requirement of real-time detection.

\section{Otsu method and its improvement}

The Otsu method can meet the real-time of system and have good effect for target segmentation, but 
single-threshold Otsu method cannot effectively segment the concealed target in PMMW body images because the images which have concealed target can be divided into background region, human body region and target region. So we extend it to double-threshold Otsu method.

\subsection{Single-threshold Otsu method}

Let the pixels of a given picture be represented in $\mathrm{L}$ gray levels $[1,2, \cdots, \mathrm{L}]$. The number of pixels at the gray level $i$ is denoted by $n_{i}$, so the total number of pixels is $N=\sum_{i=1}^{L} n_{i}$, the probability of pixel at the gray level $i$ is denoted by $p_{i}$.

$$
p_{i}=n_{i} / N, p_{i} \geq 0, \sum_{i=1}^{L} p_{i}=1
$$

In this method, the threshold operation is regarded as the partitioning of the pixels of an image into two classes by a threshold at level $t$. Then the probabilities of the first class occurrence, the class mean level and the class variance are respectively given by

$$
\omega_{0}=\sum_{i=1}^{t} p_{i}, \mu_{0}=\sum_{i=1}^{t}\left(i * \frac{p_{i}}{\omega_{0}}\right), \sigma_{0}^{2}=\sum_{i=1}^{t}\left[\left(i-\mu_{0}\right)^{2} * \frac{p_{i}}{\omega_{0}}\right]
$$

The probabilities of the second class occurrence, the class mean level and the class variance are respectively given by

$$
\omega_{1}=\sum_{i=t+1}^{L} p_{i}, \mu_{1}=\sum_{i=t+1}^{L}\left(i * \frac{p_{i}}{\omega_{1}}\right), \sigma_{1}^{2}=\sum_{i=t+1}^{L}\left[\left(i-\mu_{1}\right)^{2} * \frac{p_{i}}{\omega_{1}}\right]
$$

The total mean level of the original picture and total variance are respectively given by

$$
\mu=\sum_{i=1}^{L}\left(i * p_{i}\right), \sigma^{2}=\sum_{i=1}^{L}\left[(i-\mu)^{2} * \mathrm{p}_{i}\right]
$$

Let $\sigma_{w}^{2}, \sigma_{b}^{2}$ be the within-class variance and between-class variance respectively. An optimal threshold can be determined by maximizing one of the following criterion functions with respect to $t$ :

$$
\lambda=\frac{\sigma_{b}^{2}}{\sigma_{w}{ }^{2}}, \quad \eta=\frac{\sigma_{b}^{2}}{\sigma^{2}}, \quad \kappa=\frac{\sigma^{2}}{\sigma_{w}^{2}}
$$

Where

$$
\begin{gathered}
\sigma_{w}^{2}=\omega_{0} \sigma_{0}^{2}+\omega_{1} \sigma_{1}^{2} \\
\sigma_{b}^{2}=\omega_{0} *\left(\mu_{0}-\mu\right)^{2}+\omega_{1} *\left(\mu_{1}-\mu\right)^{2}
\end{gathered}
$$

Of the above three criterion functions, $\eta$ is the simplest and $\sigma^{2}$ is independent of $t$. Thus, the optimal threshold $t^{*}$ is

$$
t^{*}=\underset{t=1 \ldots L}{\operatorname{argmax}} \sigma_{b}^{2}
$$

Because $\sigma^{2}$ is independent of $t$ and the following basic relation always holds:

$$
\sigma_{b}^{2}+\sigma_{w}^{2}=\sigma^{2}
$$

So the function (6) is equivalent to following function:

$$
t^{*}=\underset{t=1 \ldots L}{\operatorname{argmin}} \sigma_{w}^{2}=\omega_{0} \sigma_{0}^{2}+\omega_{1} \sigma_{1}^{2}
$$

\subsection{Double-threshold otsu method}

Double-threshold Otsu method is the improvement of single-threshold Otsu method. In this method, the threshold operation is regarded as the partitioning of the pixels of an image into three classes by two thresholds at level $T_{1}$ and $T_{2}$.

The probabilities, the class mean level and the class variance of each class are respectively given by

$$
\begin{aligned}
& \omega_{0}=\sum_{i=1}^{T_{1}} p_{i} \\
& \mu_{0}=\sum_{i=1}^{T_{1}}\left(i * \frac{p_{i}}{\omega_{0}}\right) \\
& \sigma_{0}^{2}=\sum_{i=1}^{T_{1}}\left[\left(i-\mu_{0}\right)^{2} * \frac{p_{i}}{\omega_{0}}\right] \\
& \omega_{1}=\sum_{i=T_{1}+1}^{T_{2}} p_{i} \\
& \mu_{1}=\sum_{i=T_{1}+1}^{T_{2}}\left(i * \frac{p_{i}}{\omega_{1}}\right) \\
& \sigma_{1}^{2}=\sum_{i=T_{1}+1}^{T_{2}}\left[\left(i-\mu_{1}\right)^{2} * \frac{p_{i}}{\omega_{1}}\right] \\
& \omega_{2}=\sum_{i=T_{2}+1}^{L} p_{i} \\
& \mu_{2}=\sum_{i=T_{2}+1}^{L}\left(i * \frac{p_{i}}{\omega_{2}}\right) \\
& \sigma_{2}^{2}=\sum_{i=T_{2}+1}^{L}\left[\left(i-\mu_{2}\right)^{2} * \frac{p_{i}}{\omega_{2}}\right]
\end{aligned}
$$

The optimal threshold $T_{1}$ and $T_{2}$ are:

$$
T_{1}^{*}, T_{2}^{*}=\underset{1 \leq T_{1}<T_{2} \leq L}{\arg \min } \sigma_{f}^{2}=\omega_{0} \sigma_{0}^{2}+\omega_{1} \sigma_{1}^{2}+\omega_{2} \sigma_{2}^{2}
$$




\section{Double-threshold Otsu method based on prior area}

In a PMMW body image, concealed target account for small proportion compared with the human body and background, and the target will be undetected by single-threshold Otsu method. To solve this problem, we proposed double-threshold Otsu method based on prior area information to achieve the detection of small target.

The basic idea of target detection algorithm based on prior area information is describe as follows. Firstly, design objective function according to the area information of each region of PMMW image. Secondly, obtain the segmentation threshold which minimizing the objective function in accordance with histogram of PMMW image. Finally, use this threshold to segment the image and use the gray information of target to extract the target region.

Objective function of the algorithm is defined as:

$$
\sigma_{f}^{2}=\alpha_{0} \omega_{0} \sigma_{0}^{2}+\alpha_{1} \omega_{1} \sigma_{1}^{2}+\alpha_{2} \omega_{2} \sigma_{2}^{2}
$$

Where, $\alpha_{0}+\alpha_{1}+\alpha_{2}=1, \omega_{0}+\omega_{1}+\omega_{2}=1$, the meaning of $\omega_{0}, \sigma_{0}^{2}, \omega_{1}, \sigma_{1}^{2}, \omega_{2}, \sigma_{2}^{2}$ are same as the second portion, $\alpha_{0}, \alpha_{1}, \alpha_{2}$ are correction factor.

Let the area ratio of the region which at low gray levels is about $P_{0}$, the area ratio of the region which at high gray levels is about $P_{2}$, the others is about $P_{1}$. Because of adding to the prior information, it is assumed that the pixel values of image are independent of each other and to obey uniform distribution of [ [ 1256$]$ to simplify the calculation.

$$
\begin{gathered}
\omega_{0}=\frac{T_{1}}{256} \quad \mu_{0}=\frac{T_{1}}{2} \quad \sigma_{0}^{2}=\frac{T_{1}^{2}}{12} \\
\omega_{1}=\frac{T_{2}-T_{1}}{256} \quad \mu_{1}=\frac{T_{2}+T_{1}}{2} \quad \sigma_{1}^{2}=\frac{\left(T_{2}-\mathrm{T}_{1}\right)^{2}}{12} \\
\omega_{2}=\frac{L-T_{2}}{256} \quad \mu_{2}=\frac{L+T_{2}}{2} \quad \sigma_{2}^{2}=\frac{\left(L-T_{2}\right)^{2}}{12}
\end{gathered}
$$

Where $\mathrm{L}=256$, then objective function can be expressed as:

$$
\sigma_{f}^{2}=\frac{\alpha_{0} T_{1}^{3}+\alpha_{1}\left(T_{2}-T_{1}\right)^{3}+\alpha_{2}\left(256-T_{2}\right)^{3}}{256^{*} 12}
$$

Derivative and make it equal to zero:

$$
\frac{\partial \sigma_{f}^{2}}{\partial T_{1}}=\frac{\alpha_{0} T_{1}^{2}-\alpha_{1}\left(T_{2}-T_{1}\right)^{2}}{256 * 4}=0
$$

$$
\frac{\partial \sigma_{f}^{2}}{\partial T_{2}}=\frac{\alpha_{1}\left(T_{2}-T_{1}\right)^{2}-\left(1-\alpha_{0}-\alpha_{1}\right)\left(256-T_{2}\right)^{2}}{256 * 4}=0
$$

By $p_{0}=\frac{T_{1}}{256}, p_{1}=\frac{T_{2}-T_{1}}{256}$ and $p_{2}=\frac{256-T_{2}}{256}$, can be deduced

$$
\begin{gathered}
\alpha_{0}=\frac{P_{1}^{2} P_{2}^{2}}{P_{0}^{2} P_{1}^{2}+P_{1}^{2} P_{2}^{2}+P_{0}^{2} P_{2}^{2}} \\
\alpha_{1}=\frac{P_{0}^{2} P_{2}^{2}}{P_{0}^{2} P_{1}^{2}+P_{1}^{2} P_{2}^{2}+P_{0}^{2} P_{2}^{2}} \\
\alpha_{2}=1-\alpha_{0}-\alpha_{1}=\frac{P_{0}^{2} P_{1}^{2}}{P_{0}^{2} P_{1}^{2}+P_{1}^{2} P_{2}^{2}+P_{0}^{2} P_{2}^{2}}
\end{gathered}
$$

When $\alpha_{0}, \alpha_{1}, \alpha_{2}$ taken (22), (23), (24), the double-threshold segmentation algorithm can obtain the optimal threshold.

\section{Concealed target detection algorithm for PMMW image and experiment results}

Otsu algorithm based on prior area information for concealed target detection in PMMW images is mainly divided into three steps: image de-noising, image segmentation and concealed target detection. Firstly, we choose the median filter to de-nose PMMW image to meet the requirement of real-time of system and maintain the image edge as possible. Then estimate the area of each region of the image according to the parameters of the imaging system such as the field of view, perspective, imaging distance, and use Otsu method based on prior area information to segment the PMMW image. Finally, extract the target by the gray scale range of the target occupies in PMMW image to achieve the purpose of detection. Flow chat of the algorithm is as Figure 1.

In order to verify the stability and effectiveness of the target detection algorithm based on prior area information, we use the PMMW images which imaging by PMMW imaging system to simulation. The simulation results are compared with single-threshold and double-threshold Otsu algorithm. The experimental results are shown in Figure 2-4.

According to the field of view and imaging distance of the PMMW imaging system, we can estimate the human region account for about 0.35 of the whole area of 
the PMMW image, target region account for about 0.02 of the whole area of the PMMW image.

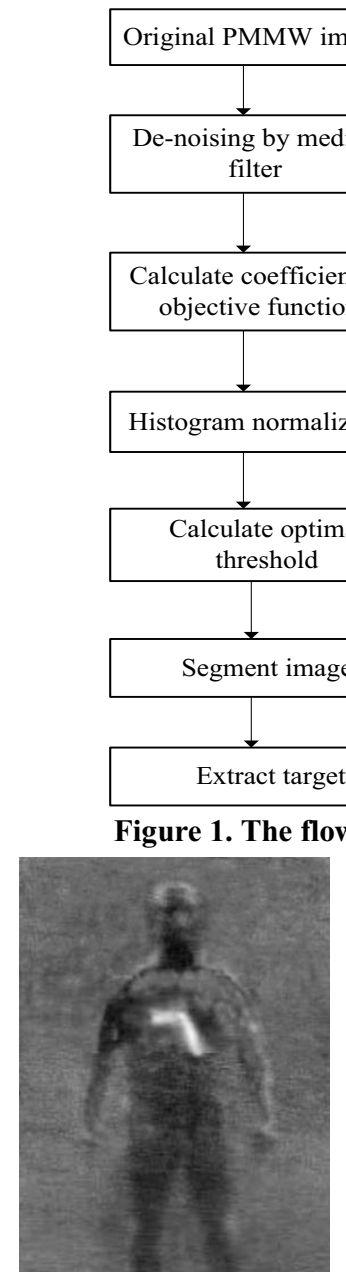

(a) PMMW Image

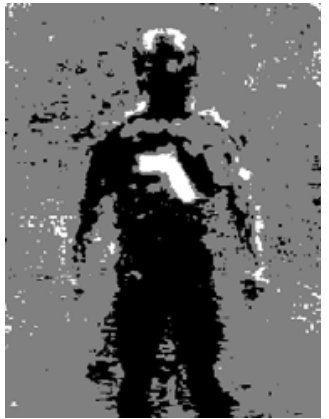

(c) The Segmentation Result of Double-Threshold Otsu (b) The Segmentation Result of Single-Threshold Otsu

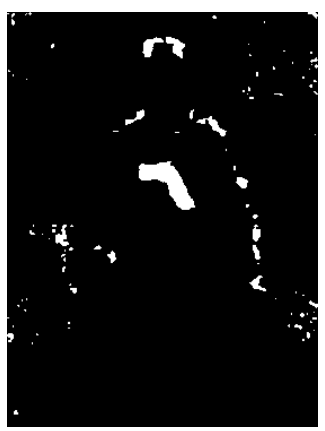

(d) The Detection Result of Double-Threshold Otsu

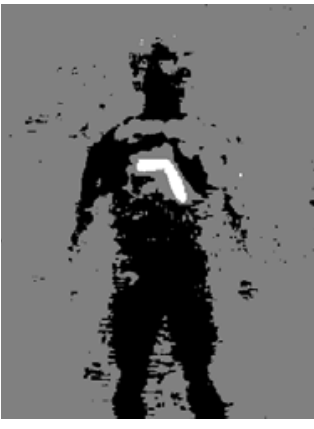

(e) The Segmentation Result of The

Proposed Algorithm

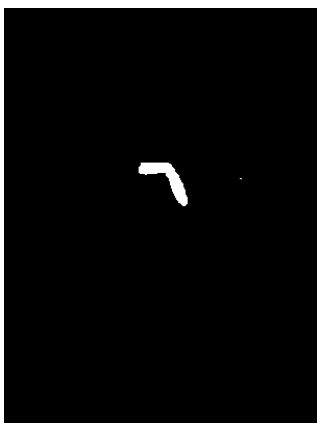

(f) The Detection Result of The Proposed Algorithm

Figure 2. Target Detection results of PMMW image

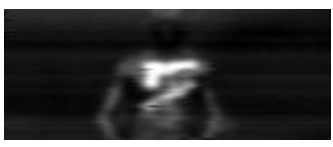

(a) PMMW Image

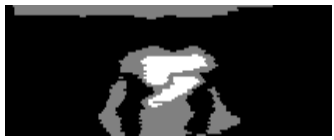

(c) The Segmentation Result of

Double-Threshold Otsu

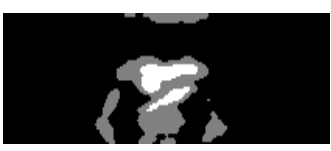

(e) The Segmentation Result of The

Proposed Algorithm

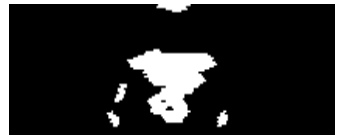

(b) The Segmentation Result of Single-Threshold Otsu

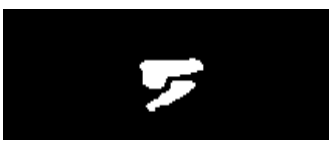

(d) The Detection Result of Double-Threshold Otsu

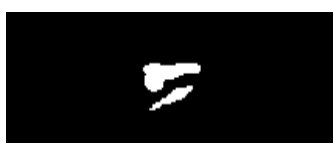

(f) The Detection Result of The Proposed Algorithm
Figure 3. Target Detection results of PMMW image

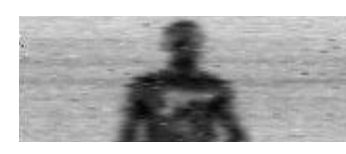

(a) PMMW Image

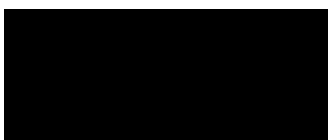

(c) The Detection Result of Double-Threshold Otsu

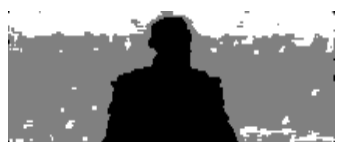

(b) The Segmentation Result of Double-Threshold Otsu

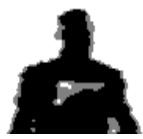

(d) The Segmentation Result of The Proposed Algorithm

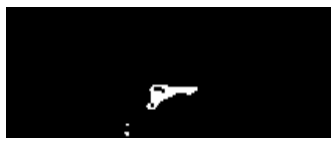

(e) The Detection Result of The Proposed Algorithm

Figure 4. Target Detection results of PMMW image

In Figure 1, we calculate coefficient by formula (22), (23), (24), normalized probability and calculate threshold by formula (11), (12), (13), (15). 
It can be seen from the simulate experiments that the Otsu algorithm based on prior area information for target detection have a best effect. Although the double-threshold Otsu algorithm has certain effect, when the target is small as seen in Figure 4(b) the target is not detected. The Otsu algorithm based on prior area information for target detection we proposed makes good use of the area information of each region, which greatly improves the detection accuracy of the algorithm. The more precise of the prior area information, the higher detection accuracy we can obtain. For the complexity of the algorithm, the algorithm proposed in this paper can meet the requirement of real-time.

\section{Conclusion}

A novel Otsu method based on prior area information for concealed target detection in PMMW images is proposed in this paper, which can obtain a high performance on concealed target detection of PMMW images. The proposed algorithm estimates the area of each region of the image according to the parameters of the imaging system such as the field of view, perspective, imaging distance and makes good use of the area information to obtain a high quality of the target detection. The experimental results show that the proposed algorithm can achieve concealed target detection of the PMMW images, and has good adaptability and stability. The algorithm is practical and has higher real-time performance compared with the traditional Otsu methods.

\section{References}

1. L. Yujiri, M. Shoucri and P. Moffa. Passive millimeter wave imaging, Microwave Magazine, IEEE, 4, 39-50(2003)

2. Haworth, D. Christopher, G. Gonzalez, ect.. Image analysis for object detection in millimetre-wave images, Passive Millimetre-wave and Terahertz Imaging and Technology, 5619, 117-128(2004)

3. XL. Shen, CR. Dietlein, ect.. Detection and Segmentation of Concealed Objects in Terahertz Images, IEEE Transactions on Image Processing, 17, 2465-2475(2008)

4. Lee, DS, Yeom, S, ect.. Real-time computational processing and implementation for concealed object detection, Optical Engineering, 51(2012)

5. W. Dai, W. Mei and XD. Zhao. Detetion and Segmentation of Concealed Objects in X-ray Compton Backscatter Images, International Conference on Image and Graphics, 175-179(2013)

6. O. Martínez, L. Ferraz, ect.. Concealed Object Detection and Segmentation over Millimeter Waves Images, Computer Vision and Pattern Recognition Workshops (CVPRW), (2010)

7. N. Otsu. A Threshold Selection Method from Gray-Level Histograms, IEEE Transactions on Systems, Man, and Cybernetics, (1979)

8. P.K.SAHOO, S.SOLTANI, and A.K.C.WONG. A Survey of Thresholding Techniques, Computer Vision, Graphics, and Image Processing, (1988) 